This is an accepted manuscript before typesetting. The final version of this manuscript in now published in Nature Geoscience available at https://doi.org/10.1038/s41561-021-00737-w. Please feel free to contact any of the authors; we welcome feedback. 


\section{A UK perspective on tackling the geoscience racial diversity crisis in 2 the Global North.}

Natasha Dowey ${ }^{1 *}$, Jenni Barclay², Ben Fernando ${ }^{3}$, Sam Giles ${ }^{4}$, Jacqueline Houghton ${ }^{5}$, Christopher Jackson ${ }^{6}$, Anjana Khatwa ${ }^{7}$, Anya Lawrence ${ }^{4}$, Keely Mills ${ }^{8}$, Alicia Newton ${ }^{9}$, Steven Rogers ${ }^{10}$ and Rebecca Williams $^{11}$

${ }^{1}$ Sheffield Hallam University, UK; ${ }^{2}$ University of East Anglia, UK; ${ }^{3}$ University of Oxford, UK; ${ }^{4}$ University of Birmingham, UK; ${ }^{5}$ University of Leeds, UK; ${ }^{6}$ University of Manchester, UK; ${ }^{7}$ Wessex Museums, UK; ${ }^{8}$ British Geological Survey, UK; ${ }^{9}$ Geological Society of London, UK; ${ }^{10}$ Keele University, UK; ${ }^{11}$ University of Hull, UK *Corresponding Author: N.Dowey@shu.ac.uk

Geoscientists will play key roles in the grand challenges of the twenty-first century, but this requires our field to address its past when it comes to diversity and inclusion. Considering the bleak picture of racial diversity in the UK, we put forward steps institutions can take to break down barriers and make the geosciences equitable.

The roots of modern geoscience lie in early colonial principles that land could belong to those willing to use its products, regardless of indigenous territories and practices. The production of geoscience knowledge has therefore been historically tied to a desire to explain the distribution and extractability of resources, largely for the benefit of the colonising force ${ }^{1}$. This knowledge now has an essential role to play in equitable and sustainable development, but it cannot be successfully applied without diverse representation amongst geoscientists. Addressing global problems that impact people from all walks of life means we must work within and across a wide array of communities.

A robust approach to diversity and inclusion needs to begin at home, especially in the very countries that have benefited from the structures and wealth of a colonial past. Geoscience in the Global North is disproportionately white, a result of both historic systemic racism that impacts academia as a whole ${ }^{2}$ and subject-specific issues that make our discipline less inclusive to many underrepresented groups ${ }^{3}$. In the USA, for example, just $6 \%$ of Geoscience doctorate degrees are awarded to students from underrepresented minorities (defined as American Indian or Alaska Native, Black or African American, Hispanic or Latino) despite 31\% 
hostile environments that have led to inequality in our discipline, attract researchers from a variety of backgrounds and retain them throughout their careers.

The lack of diversity in geoscience has been documented in North America ${ }^{4,5}$, but there has been little focus on diversity trends in postgraduate geoscience research ('PGR'; PhD and MRes courses) in other regions of the Global North. This work aims to highlight issues facing UK Geoscience in a similar way to Bernard \& Cooperdock ${ }^{4}$ in the US, to give international perspective to these discussions. The data we present from the UK Higher Education Statistics

To provide context for our discussion, we must reflect on our own experiences. Of the twelve authors of this paper, four identify as BAME (we use the term 'BAME' for Black, Asian and minority ethnic throughout this piece for consistency with HESA terminology, but recognise this homogenises different identities and obscures experiences felt by one race or ethnicity). The majority of us have not been the victim of direct racism. We approach this from the perspective of concerned Geoscience academics, rather than scholars in equity, diversity and inclusion (EDI), although a number of us have EDI responsibilities in institutions or charities. Our aim is to

54 highlight the situation and promote the need for action. Geoscientists in both industry and academia should work together to listen to diverse voices, challenge biases and transform geoscience culture to be more inclusive and accountable.

\section{Factors involved in racial inequity in UK Geoscience}

\section{Pre-university}

Fundamental lack of acknowledgement that geoscience is deeply rooted in, and built on, colonialism, white power, violence, exploitation and slavery pervades relationships in the present and is a barrier to forging equitable partnerships ${ }^{3}$. The stereotype of a geoscientist as a white man, compounded by the perception that geoscience is an outdoors only activity (and perpetuated by marketing materials that feature white students in rugged backgrounds), is particularly discouraging to those from minority ethnic backgrounds.

In a recent Geological Society of London survey, $60 \%$ of undergraduate Geology students mentioned a lifelong interest in the natural environment. Natural environments are less 
accessible to children from urban settings (which are more ethnically diverse; over $98 \%$ of Black African, Pakistani and Bangladeshi people live in urban locations in the $\mathrm{UK}^{8}$ ) and children from low-income households (more likely to be Pakistani, Bangladeshi, Chinese or Black than white in the $\left(K^{9}\right)$. The UK Department for Environment, Food and Rural Affairs report that $18 \%$ of children living in the most deprived areas never visit the countryside, with Black and Asian

74 families the least likely group to visit rural areas.

Furthermore, a career in postgraduate geoscience research may not be seen to offer the 78 financial security of other professions, such as Medicine, by some communities ${ }^{10}$.

80

\section{Retention into postgraduate research and beyond}

BAME students applying to high-tariff, research-intensive, institutions are less likely to be accepted than white students with comparable qualifications. For example, BAME applicants to Mathematical, Physical and Life Sciences subjects at Oxford are 5.8\% less likely to receive an offer than their white counterparts, even after accounting for prior attainment and course choice $^{11}$. In 2018/19, Black students made up just 3.9\% of students at high-tariff universities, compared to $12.2 \%$ at low-tariff universities ${ }^{6}$. Once at university, BAME students are less likely to gain a first or 2:1 degree classification than their white peers. A range of factors have been proposed to explain this but an unexplained gap still exists, likely due to unconscious bias and inequitable frameworks within HE that disadvantage Black and minority ethnic students ${ }^{12}$.

Aspects of the $\mathrm{PhD}$ application process, such as preference for high-tariff university graduates and selection using metrics that reflect access and resource availability more than student achievement, disproportionately detriment marginalised and underrepresented students ${ }^{13}$. Just $9 \%$ of UKRI (UK national funding body) studentships were awarded to ethnic minorities in 2018/1914; a dismal statistic considering that $19.4 \%$ of $18-34$ year olds identify as BAME ${ }^{15}$. These numbers are even lower for NERC (UK national funder of natural science), with just $6 \%$ of studentships going to ethnic minorities ${ }^{14}$. Having role models to identify with is important to foster a sense of belonging in the scientific community; a lack of BAME representation at faculty level has been linked to BAME students not continuing to PGR. Across the UK just $10.8 \%$ of professors are BAME ${ }^{16}$, but of the 2,390 staff working in Earth, Marine and Environmental Sciences in 2018/19 only $90(3.9 \%)$ were BAME; the second lowest figure of all Science, Engineering and Technology disciplines ${ }^{17}$. This 
'institutional whiteness' can result in feelings of isolation, and the few BAME staff present being relied upon to be representative of all BAME issues and burdened with advancing equality without meaningful reward.

The geosciences have additional subject-specific hostile environments ${ }^{3}$ that may deter BAME students from continuing in PGR. Fieldwork requirements create barriers to ethnic minorities, for reasons including cultural sensitivity (e.g. co-ed residential trips), cost, inclusivity and racial

Note that some of the above issues are intersectional; BAME students may experience overlapping barriers depending on their gender, sexuality, disability, class, or nationality ${ }^{20}$, particularly in the field. Building a culture more inclusive to BAME students can broaden participation to a range of minority groups.

\section{What can we do about it?}

\section{Decolonisation}

121 There has been a growing demand for the academy to contend with its colonial links and 122 institutional whiteness ${ }^{21}$. In geoscience we reference the likes of Adam Sedgwick and Henry de 123 la Beche in our teaching but rarely mention their links to slavery, which are now being 124 recognised. We teach mapping, surveying, and mining geology but we rarely explain how these 125 activities link to the growth of the British Empire ${ }^{1}$, or modern destruction of indigenous sites.

126 Moving forward geoscientists must reflect and engage with social scientists and historical 127 scientists to explore these links, teaching them through the positive lens of geoethics ${ }^{22}$.

The relationship of field-based disciplines with the land has typically taken a colonial approach, 130 of white, western field scientists visiting a location, removing samples (often with the help of 131 local people), then extricating this knowledge and publishing it in paywalled, western journals 132 (often without local co-authors). This work may disrespect the customs and beliefs of indigenous 133 communities ${ }^{23}$. In a study focussed on First Nation communities, Datta ${ }^{24}$ explains successful, 134 sustainable 'land-based education', which understands the land as dynamic, as relational (e.g. 135 spiritual), and as linked to well-being. We can learn from studies like this and be more cognizant 
136 of the cultural backgrounds of both our field sites and students during fieldwork, particularly if

137 work is conducted in the Global South or indigenous lands.

\section{Inclusive teaching}

140 Geoscience is vital in developing a more sustainable society, and a critical aspect of sustainable

141 development is the reduction of inequalities (Goal 10, UN Sustainable Development Goals).

142 Sustainability in Geography, Earth and Environmental Science HE education is considered by

143 Gormally (2019), who advocates for interdisciplinarity, diversity of approach, and moving

144 beyond environmental sustainability to include social, cultural and political perspectives.

146 By teaching a geoscience curriculum more focussed on global perspectives of sustainability,

147 and less on (typically white) traditional geoscience perspectives, we can create a more relevant 148 and inclusive curriculum to students of all races and ethnicities ${ }^{25}$.

\section{Representation}

We can invest resources in racially diverse promotional materials and ambassador schemes that reward outreach work ${ }^{4}$ and do not disproportionately place the burden on BAME students ${ }^{26}$. We can also support grass-roots initiatives to amplify BAME voices in geoscience (e.g. Black In Geoscience and Black Geographers), and invite diverse Geoscience researchers to deliver departmental seminars and showcase innovative science. Crucially, we must increase the diversity of our faculty staff, by implementing BAME staff development opportunities (like StellarHE) to counterbalance structural racism, mitigating underrepresentation and facilitating career progression.

\section{Subject awareness}

161 By working further back along the student lifecycle, we can make it easier for those from BAME 162 communities to connect with nature. Natural heritage organisations need to work closely with community leaders to welcome and nurture positive experiences for BAME children and young people in green spaces. Black2Nature camps run by youth campaigner and environmentalist Mya-Rose Craig have opened pathways that have enabled young people from deprived areas in Bristol to learn about birding, conservation and wildlife; universities can play a part in similar 167 activities through outreach.

\section{Removing barriers}


170 A variety of practical steps can be taken to make fieldtrips more inclusive for ethnic minority

171 students. By fully subsidising trips and equipment costs departments can remove barriers to

172 students from low-income backgrounds. To ensure students feel safe from discrimination, field

173 leaders can incorporate recommendations laid out by Anadu and others ${ }^{19}$, including racial risk

174 assessments, antidiscrimination and allyship training, and full documentation of race-related

175 incidents. Trips should be developed with a careful focus on the skills and learning outcomes

176 needed for modern geoscience employment, with mitigations in place to allow all students to

177 achieve them. Professional bodies should reform accreditation requirements around mandatory

178 days in the field. We can create a more inclusionary space by promoting positive accounts and

179 perspectives of fieldwork from minority groups, challenging and disrupting the dominant white,

180 male image of fieldwork.

182 Ring-fenced opportunities, such as funded research experiences, summer schools, internships, 183 and studentships, are clear and evidenced pathways to increased chances of progression for 184 underrepresented groups ${ }^{27}$. Working collaboratively with schools, colleges and other universities 185 can make such initiatives more viable and increase their reach.

We can hold funding organisations and institutions accountable for transparency in their recruitment processe ${ }^{13}$ and form interview panels that understand these barriers, helping ensure improved diversity in successful applicants ${ }^{27}$. We can push for the publication of candidate demographic data at application, interview, offer and acceptance stages, to provide a clearer picture of postgraduate recruitment diversity ${ }^{13}$.

\section{Effective, long-lived initiatives}

194 Initiatives do not end at recruitment. Allocating more resources to training in equity and inclusion, and creating more 'champions' of diversity to support the interests of minority groups and encourage reflection within Geoscience departments, would be a significant step forward in removing hostile environments.

To ensure our efforts are effective and long-lived, we must submit funding bids for evidencedriven action research that works to address data gaps, advocates for real change, and develops strategies to broaden participation. We can work with other subjects and bodies facing similar challenges, sharing transferable solutions across the HE sector. 
204 Crucially, we need to acknowledge the hostile environments that deter BAME students from 205 both applying to, and continuing with, our discipline. These problems are real and felt by 206 many $^{28}$. We must address personal and structural biases, and go beyond this to be actively anti207 racist. The less diverse a field is, the more prevalent implicit biases become ${ }^{5}$. We must act now, 208 and have those difficult conversations, to create a modern geoscience research culture that 209 reflects the diverse nature of the planet we study.

Acknowledgements: Many thanks to Catherine Souch of the Royal Geographical Society, 212 Liam Herringshaw, Lis Gallant and three Nature reviewers for positive and constructive 213 feedback on this piece.

215 The authors declare no competing interests.

\section{Box 1 - The data}

218 While the absolute number of UK-domiciled students who identify as BAME (defined as 'Black', 219 'Asian', 'Mixed' and 'Other' in UK Census and HESA ethnicity data) in UK Higher Education 220 (HE) has grown by $>150,000$ since 2003, there remain pronounced disparities between white and BAME students in their retention into postgraduate research ${ }^{29}$. These disparities vary between disciplines, and between ethnic groups within the BAME identifier.

Physical Geography, Geology and Environmental Sciences are the three worst Physical Science subjects for BAME student undergraduate participation in UK HE, and are very poor for retention of these students into PGR ${ }^{6}$. In the 2011 UK Census, $18.5 \%$ of UK $18-24$ year olds were from Black, Asian or Minority Ethnic backgrounds ${ }^{15}$. However, just $5.2 \%$ of Physical Geography, $6.86 \%$ of Environmental Science and $10.4 \%$ of Geology PGR students identified as BAME in 2018/19. These statistics are far lower than Physical Science subjects with the highest BAME PGR representation (22.5\% in Materials Science and 14.8\% in Chemistry). On average, over the past 5 years just $1.4 \%$ of Geology PGR students were Black ${ }^{6}$, even though 3.8\% of UK 18-24 year olds identify as Black ${ }^{15}$. During the last five years, there have been two years for both Geology and Physical Geography when no Black women took up full time PGR study. Retention of BAME Physical Geography and Environmental Science students into PGR was worse in 2018/19 than over the five years from 2014/15 to 2018/19 (mean averages shown in figure); the situation is not improving with time ${ }^{6}$. 


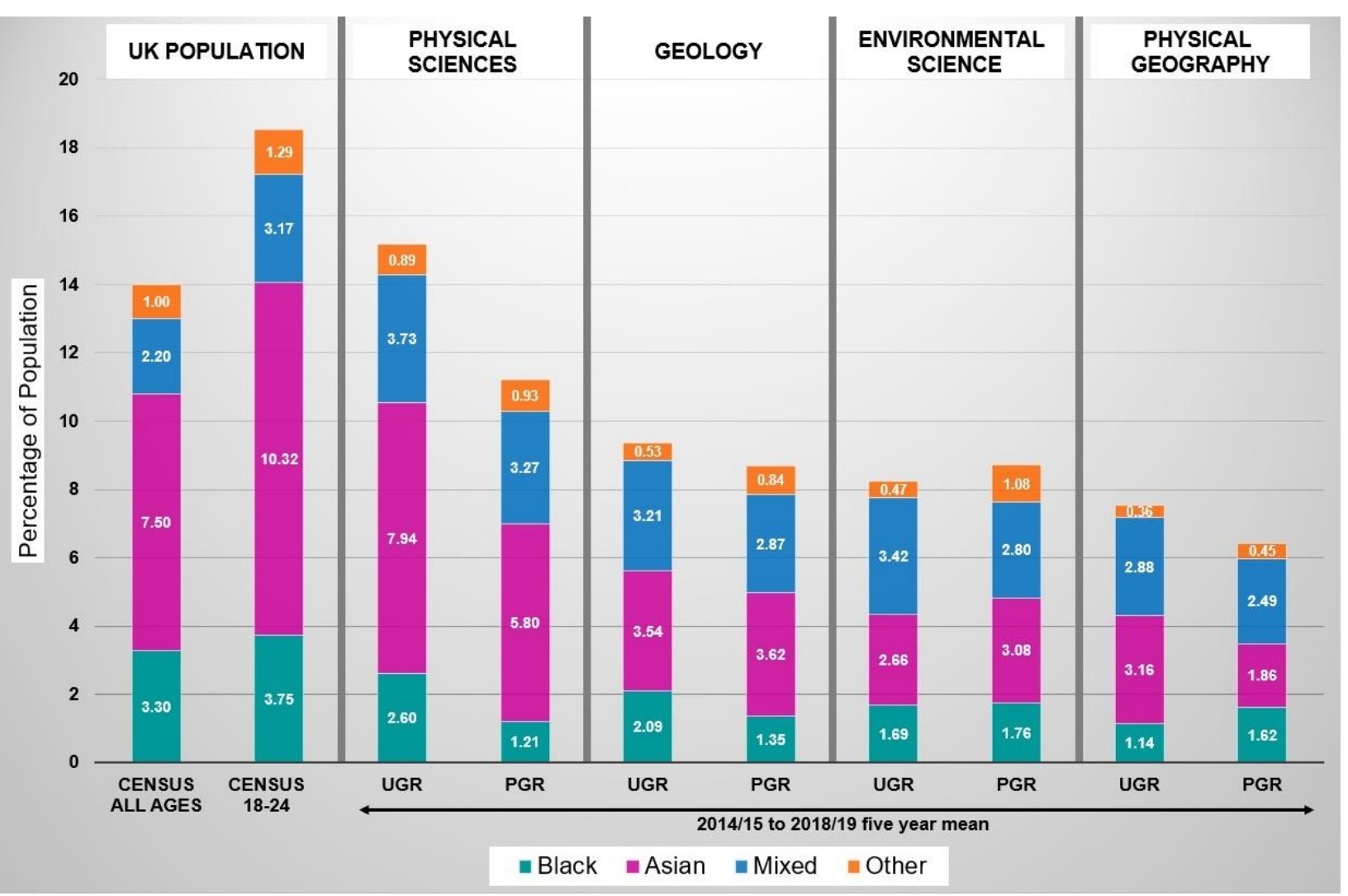

Representation of BAME (Black, Asian, Mixed and Other ethnic minorities) students in Physical Sciences, Geology, Environmental Science ('Science of Aquatic \& Terrestrial Environments') and Physical Geography ('Physical

Geographical Sciences') from Higher Education Statistics Agency data ${ }^{6}$, alongside ethnicity data from the 2011 UK Government Census ${ }^{15}$. HESA data are based on full-time "all undergraduate" (UGR) and full-time "postgraduate research" (PGR) categories and are a five-year mean average of data from 2014/15 to 2018/19.

\section{Notes on the data}

HESA publish 'subject of study by ethnicity' data broken down by level of study for 2014/15 to $2018 / 19$ on their website ${ }^{6}$. Data from $1994 / 95$ to $2014 / 15$ are available ${ }^{30}$, but are not broken down by type of postgraduate study (research versus taught), or fully by ethnicity ("Mixed" and "Other" ethnic categories are grouped); this archive data is therefore not used here. We use the term "geoscience" here to group Physical Geography, Geology and Environmental Science (due to HESA categories), but recognise our recommendations are applicable to a variety of allied disciplines. Although we present quantitative data up front, we acknowledge that qualitative studies (some of which we reference herein), voices and insights are vital in this discussion.

\section{Key References (see Supplementary Materials for full reading list)}


257 1. Stafford, R. A. Geological Surveys, Mineral Discoveries, and British Expansion, 1835-71. 258 J. Imp. Commonw. Hist. 12, 5-32 (1984).

259 2. Gillborn, B. D. Education policy as an act of white supremacy: Whiteness, critical race 260 theory and education reform. Journal of Education Policy vol. 20 485-505 (2005).

261 3. Marín-Spiotta, E. et al. Hostile climates are barriers to diversifying the geosciences. Adv. 262 Geosci. 53, 117-127 (2020).

263 4. Bernard, R. E. \& Cooperdock, E. H. G. No progress on diversity in 40 years. Nat. Geosci. 264 11, 292-295 (2018).

265 5. Dutt, K. Race and racism in the geosciences. Nat. Geosci. 13, 2-3 (2020).

266 6. Higher Education Statistics Authority. Personal Characteristics by Subject of Study. https://www.hesa.ac.uk/data-and-analysis/students/what-study/characteristics (2019).

10. Fernando, B. \& Antell, G. Recommendations for improving racial equality, diversity, and inclusion in the Department of Earth Sciences, University of Oxford. (2020).

11. Inge, S. Scale of Oxford's 'white bias' laid bare by internal report. Research Professional News (2020).

12. Leading Routes. The Broken Pipeline. https://leadingroutes.org/mdocs-posts/the-brokenpopulation-by-ethnicity/national-and-regional-populations/regional-ethnic-diversity/latest

9. Office for National Statistics. Child poverty and education outcomes by ethnicity. https://www.ons.gov.uk/economy/nationalaccounts/uksectoraccounts/compendium/econo micreview/february2020/childpovertyandeducationoutcomesbyethnicity\#child-povertyand-ethnicity (2020). pipeline-barriers-to-black-students-accessing-research-council-funding (2019).

13. Giles, S. et al. Open letter concerning CDT and DTP student recruitment across the UK. https://docs.google.com/document/d/1EInAKFI7px2DxYv-sAZxVOyTjkmAE29CzFFnjxw6hg/edit (2020).

14. UK Research and Innovation. Diversity Results for UKRI funding data 2014/15 to 201819. https://www.ukri.org/files/about/ukri-diversity-report/ (2020).

15. GOV.UK. Population of England and Wales. https://www.ethnicity-factsfigures.service.gov.uk/uk-population-by-ethnicity/national-and-regionalpopulations/population-of-england-and-wales/latest (2011). 
16. Higher Education Statistics Authority. Higher Education Staff Data. https://www.hesa.ac.uk/data-and-analysis/staff (2019).

17. AdvanceHE. Staff Statistical Report 2019. https://www.advance-he.ac.uk/knowledgehub/equality-higher-education-statistical-report-2019 (2019).

18. Giles, S., Jackson, C. \& Stephen, N. Barriers to fieldwork in undergraduate geoscience degrees. Nat. Rev. Earth Environ. 1, 77-78 (2020).

19. Anadu, J., Ali, H. \& Jackson, C. Ten Steps to Protect BIPOC Scholars in the Field. Eos

20. Crenshaw, K. Demarginalizing the Intersection of Race and Sex: A Black Feminist Critique of Antidiscrimination Doctrine, Feminist Theory and Antiracist Politics. Univ. Chic. Leg. Forum 1989, (1989).

21. Peters, M. A. Why is My Curriculum White? Educ. Philos. Theory 47, (2015).

22. Di Capua, G., Peppoloni, S. \& Bobrowsky, P. T. The cape town statement on geoethics. Ann. Geophys. 60, (2017).

23. Reano, D. Using Indigenous Research Frameworks in the Multiple Contexts of Research,

24. Datta, R. K. Rethinking environmental science education from indigenous knowledge perspectives: an experience with a Dene First Nation community. Environ. Educ. Res. 24, 50-66 (2018).

25. Stewart, I. S. \& Gill, J. C. Social geology - integrating sustainability concepts into Earth sciences. Proc. Geol. Assoc. 128, 165-172 (2017).

26. Gewin, V. The time tax put on scientists of colour. Nature (2020).

27. Dutt, K. Promoting Racial Diversity Through Transparency. Eos (Washington. DC). 100, (2019).

28. Ali, H. Petition - Call for a Robust Anti-Racism Plan for The Geosciences. Change.org https://www.change.org/p/geoscientists-call-for-a-robust-anti-racism-plan-for-thegeosciences?recruited_by_id=54b3bdc0-3257-11e9-83860954e4ca4466\&recruiter $=936695691$.

29. Advance HE. Equality in higher education: statistical report 2019. https://www.advancehe.ac.uk/knowledge-hub/equality-higher-education-statistical-report-2019 (2019).

30. Higher Education Statistics Authority. Publications archive. https://www.hesa.ac.uk/dataand-analysis/publications (2021). 